\title{
Association between postoperative thromboembolism prophylaxis and complications following urological surgery
}

\author{
MARIA ANGELA CERRUTO, CAROLINA D'ELIA, MARCO PICCOLI, GIOVANNI CACCIAMANI, \\ DAVIDE DE MARCHI, PAOLO CORSI, VINCENZO DE MARCO, \\ STEFANO CAVALLERI and WALTER ARTIBANI
}

Urology Clinic, Verona Integrated University Hospital, 37134 Verona, Italy

Received September 17, 2014; Accepted July 27, 2015

DOI: $10.3892 / \mathrm{etm} .2015 .2845$

\begin{abstract}
Thromboembolism represents the most significant complication and cause of non-surgical mortality in major urological surgery. The aim of the present study was to assess the association between the type of pharmacological thromboembolism prophylaxis and the postoperative complication rate in a cohort of patients undergoing major urological surgery. All consecutive patients treated with major urological surgery between December 2011 and March 2013 were evaluated. For each patient, clinical and demographic data, as well as information on the post-surgical complications and the type of pharmacological thromboembolism prophylaxis, were collected. In total, 453 patients (mean age, 63.36 \pm 12.05 years) were recruited $(43.5 \%$ for prostate surgery, $33.1 \%$ for renal surgery, $12.1 \%$ for bladder surgery and $11.3 \%$ for other surgery). Postoperative blood transfusions were required in 50 cases $(11.0 \%)$. A total of 32 patients $(7.1 \%)$ underwent re-intervention due to the occurrence of grade $\geq 3$ complications, with a readmission rate of $2.0 \%$. According to the Clavien-Dindo Classification, the complications were grade 1 in $36.0 \%$ of the cases, grade 2 in $19.4 \%$, grade 3 in $6.0 \%$, grade 4 in $2.0 \%$ and grade 5 (mortality) in $0.7 \%$. Only 1 case of deep venous thrombosis not associated with pulmonary thromboembolism was observed. Univariate analyses showed a significant negative association (higher risk of complications) between the use of $>4,000 \mathrm{IU}$ enoxaparin as the thromboembolism prophylaxis and postoperative blood transfusion rate $(\mathrm{P}=0.045)$, re-intervention rate $(\mathrm{P}=0.001)$ and the occurrence of grade $\geq 3$ complications $(\mathrm{P}<0.001)$. Multivariate analysis confirmed the significant association between the use of $>4,000$ IU enoxaparin and both re-intervention rate $(\mathrm{P}=0.013)$ and occurrence of grade $\geq 3$ complications $(\mathrm{P}=0.002)$. High doses of enoxaparin
\end{abstract}

Correspondence to: Dr Carolina D'Elia, Urology Clinic, Verona Integrated University Hospital, 10 Piazza le L.A. Scuro, 37134 Verona, Italy

E-mail: karolinedelia@gmail.com

Key words: complication, thromboembolism, prophylaxis, surgery
(>4,000 IU) may lead to an increased risk of re-intervention and severe postoperative complications following major urological surgery. Randomised, controlled trials comparing the effect of different types of pharmacological thromboembolism prophylaxis on postoperative complications following major urological surgery are required.

\section{Introduction}

Thromboembolism represents the most significant complication and cause of mortality in urological surgery (1); between 1 and $5 \%$ of patients develop a symptomatic venous thromboembolism (VTE), which is almost the same rate as that associated with major abdominal surgery (2). The highest incidence of VTE was documented by White et al (3) in patients undergoing radical cystectomy (3.7\%); however, it is important to emphasise that similar incidence rates $(3.6 \%)$ were found in oncology patients undergoing percutaneous nephrostomy placement, a minor surgical procedure.

Due to the significant incidence of thromboembolic events in urological surgery, the American Urological Association (AUA) recommends antithrombotic prophylaxis in all patients undergoing urological procedures. In minimally invasive procedures and in low-risk patients, mechanical prophylaxis or early mobilisation may be considered adequate, in order to reduce the risk of bleeding (4). The value of pharmacological prophylaxis is not, in fact, clear: The American College of Chest Physicians (ACCP) recommends its use, but the evidence is mainly derived from non-urological studies (5). With regard to urology, the epidemiological studies should into account the improvements in surgical techniques, the new prophylaxis protocols and early mobilisation, which may be responsible for a reduction in the prevalence of VTE. In a large, multicentre study of 5,951 patients undergoing laparoscopic and robotic prostatectomy, the VTE and pulmonary embolism rates were found to be 0.5 and $0.2 \%$, respectively (6). Of these patients, $67 \%$ received heparin prophylaxis, which was associated with an increased estimated intraoperative blood loss (300 vs. $200 \mathrm{ml}$ ) and higher transfusion (4.2 vs. $3.1 \%$ ) and re-intervention (1.6 vs. $0.8 \%$ ) rates.

In 2008, the AUA recommended the use of intermittent pneumatic compression devices, together with early ambula- 
tion protocols, for all procedures, including robot-assisted laparoscopic urological surgery. Furthermore, it was recommended that pharmacological prophylaxis with low-dose heparin or unfractionated heparin should be considered in patients belonging to a high-risk population (e.g. patients with previous VTE) (4).

With regard to radical nephrectomy, the guidelines recommend pharmacological prophylaxis with heparin; however, this prophylaxis should be avoided in those patients undergoing partial nephrectomy due to the bleeding risk (7).

Radical cystectomy with pelvic lymphadenectomy represents a challenge for urologists and is the gold standard treatment for non-metastatic, muscle-invasive (or high-risk non-muscle-invasive) bladder cancer (8). The complications following radical cystectomy are due, in most cases, to the intestinal resection; however, Shabsigh et al (9) documented a high incidence of thromboembolic complications $(8 \%)$ and bleeding complications, with a transfusion rate of $\sim 9 \%$. Furthermore, as described in the review of the California Patient Discharge Data Set by White et al (3), the highest incidence of postoperative thromboembolism concerns radical cystectomy, reaching a $3.7 \%$ rate. In the AUA Best Practice Statement, patients undergoing radical cystectomy and urinary diversion were considered to be high risk, and pharmacological prophylaxis was recommended, if possible, in combination with mechanical prophylaxis (4).

The aim of the present study was to analyse the correlations between the type of pharmacological prophylaxis and the early complication and re-intervention rates in a cohort of patients undergoing urological surgery between December 2011 and March 2013.

\section{Patients and methods}

Baseline data collection. The data regarding all consecutive patients undergoing major urological surgery at the Urology Clinic of the Verona Integrated University Hospital (AOUI; Verona, Italy) between December 2011 and March 2013 were prospectively collected and retrospectively reviewed. The procedures were grouped as follows: Kidney, prostate, bladder and other procedures (miscellaneous).

The demographic, clinical, pre- and perioperative characteristics were collected for each patient, including age, body mass index (BMI), smoking, comorbidities, clinical TNM stage (in the case of urological malignancy), preoperative Gleason Score and prostate-specific antigen level (in the case of prostate cancer), year of surgery, antithrombotic prophylaxis and dosage, and haemoglobin level. This study was conducted according to the Declaration of Helsinki and was approved by Verona Integrated University Hospital (Verona, Italy). All patients were informed regarding the planned use of the healthcare data.

Antithrombotic prophylaxis. All patients included in the study were supplied with elastocompressive stockings and subjected to early mobilisation and pharmacological therapy. With regard to pharmacological prophylaxis, two drugs were predominantly used: Patients undergoing surgery between December 2011 and September 2012 received dalteparin (Fragmin $^{\circledR}$; Pfizer, Inc., New York, NY, USA) subcutaneously, while patients undergoing surgery between October 2012 and March 2013 received enoxaparin (Clexane ${ }^{\circledR}$; Sanofi-Aventis, Bridgewater, NJ, USA). This change in drug type occurred as a result of supply changes by the Pharmacy of the AOUI. Calciparin ${ }^{\circledR}$ (Eparina Calcica, Italfarmaco, Milan, Italy) was administered to patients with chronic renal failure, as enoxaparin predominantly undergoes renal elimination.

Low-risk patients without major comorbidities were prescribed only the use of elastic stockings and early mobilisation; in the case of low-risk patients undergoing radical prostatectomy and robot-assisted prostatectomy, low-molecular-weight heparin (LWMH) was stopped on the same day and patients were mobilised on the evening of surgery. In patients with risk factors for thromboembolism or undergoing medium/high-risk surgery, e.g. open pelvic surgery, pharmacological prophylaxis was continued for between 2 and 4 weeks postoperatively.

The first heparin administration was performed the evening before surgery. Comorbidities were assessed using the age-adjusted Charlson Comorbidity Index (10), and the pre-operative anaesthesiological risk was classified by the American Society of Anaesthesiologists (ASA) score (11).

Intra- and postoperative variables. The following intraoperative variables were analysed: Duration of surgery, estimated blood loss, intraoperative transfusion and first operator. Data on the early complications (occurring within 90 days after surgery) were collected by reviewing the electronic and/or paper-based outpatient archives or contacting the patient. In order to perform a systematic collection, all complications were recorded, defined and graded in accordance with the Clavien-Dindo classification (12).

The following postoperative variables were evaluated: Diuresis and haemoglobin level in the first 3 postoperative days, pre-discharge haemoglobin level, volume of drainage, central venous catheter and nasogastric tube placement and removal, time to flatus and defecation, and use of parenteral therapy.

Statistical analysis. Normally distributed continuous variables are presented as the mean \pm standard deviation, while non-normally distributed variables are expressed as the median and interquartile range. The Pearson $\chi^{2}$ and Student's $t$-tests were used to compare categorical and continuous variables, respectively, and logistic regression was used to perform multivariate analysis. A two-sided P-value of $<0.05$ was considered to indicate a statistically significant difference. All statistical analyses were performed using the Statistical Package for the Social Sciences (SPSS) software, version 20.0 (IBM SPSS, Armonk, NY, USA).

\section{Results}

Clinical and demographic data. A total of 453 consecutive patients that were treated at the Urology Department of the AOUI between December 2011 and March 2013 were recruited for the study. The following types of procedure were conducted: 197 for prostate disease $(43.5 \%), 150$ for renal disease $(33.1 \%), 55$ for bladder disease $(12.1 \%)$ and 51 for other diseases (11.3\%) (Table I). 
Table I. Procedures

\begin{tabular}{lc}
\hline Procedure (n=453) & Patients, $\mathrm{n}(\%)$ \\
\hline Vesical & $55(12.1)$ \\
Radical cystectomy and ileal conduit & 27 \\
Radical cystectomy and neobladder & 24 \\
Radical cystectomy and ureterostomy & 2 \\
Enterocystoplasty & 1 \\
Bladder diverticulectomy & 1 \\
Kidney & $150(33.1)$ \\
Tumor enucleation & 51 \\
Partial nephrectomy & 4 \\
Nephrectomy & 45 \\
Robotic nephrectomy & 9 \\
Cyst enucleation & 3 \\
Nephroureterectomy & 25 \\
Robotic pyeloplasty & 9 \\
Pyeloplasty & 4 \\
Prostate & $197(43.5)$ \\
Robot-assisted radical prostatectomy & 99 \\
Radical retropubic prostatectomy & 74 \\
Millin's retropubic prostatectomy & 4 \\
Miscellaneous & $51(11.3)$ \\
\hline
\end{tabular}

Table II. Clinical, intra- and postoperative data.

\begin{tabular}{lcc}
\hline Parameter & Range & Mean \pm SD \\
\hline Age (years) & $16-88$ & $63.36 \pm 12.05$ \\
BMI $\left(\mathrm{kg} / \mathrm{m}^{2}\right)$ & $18-44$ & $26.23 \pm 3.80$ \\
Cigarettes (n/day) & $0-60$ & $2.24 \pm 6.17$ \\
Antibiotic prophylaxis (POD) & $0-64$ & $9.14 \pm 5.55$ \\
Charlson score & $0-11$ & $3.49 \pm 1.73$ \\
Duration of surgery (min) & $20-430$ & $159.08 \pm 68.57$ \\
Flatus (POD) & $0-11$ & $2.19 \pm 0.98$ \\
Defecation (POD) & $1-11$ & $3.45 \pm 1.23$ \\
Mobilisation (POD) & $1-11$ & $1.38 \pm 1.03$ \\
Drainage (cm $\left.{ }^{3}\right)$ & $0-11,095$ & $740.62 \pm 1,149.97$ \\
Feeding (POD) & $1-11$ & $2.00 \pm 1.30$ \\
Discharge (POD) & $2-84$ & $8.85 \pm 6.91$ \\
\hline
\end{tabular}

BMI, body mass index; POD, postoperative days; SD, standard deviation.

The mean age of the patients was $63.36 \pm 12.05$ years, and the distribution of the patients between the $<65$ years $(47.5 \%)$ and $\geq 65$ years $(52.5 \%)$ age groups was almost equal. A total of $21.6 \%$ of the patients were female (98/453), and the mean BMI was $26.23 \pm 3.80 \mathrm{~kg} / \mathrm{m}^{2}$. The mean age-adjusted Charlson score was $3.49 \pm 1.73 ; 18.1 \%$ of the patients were classed as smokers and $79.7 \%$ presented comorbidities (Table II). The preoperative ASA score was as follows: ASA I in 37 cases (8.2\%), ASA II in 306 (67.5\%), ASA III in 105 (23.1\%) and ASA IV in 5 (1.1\%).

Intra- and postoperative data. Table III shows the types and dosages of pharmacological prophylaxis utilised in the present study. A total of $76.2 \%$ (345/453) of the patients underwent
Table III. Heparin prophylaxis.

\begin{tabular}{lcr} 
A, Kidney procedures $(\mathrm{n}=150)$ & & \\
\hline Prophylaxis & Doses (IU) & $\mathrm{N}$ \\
\hline Dalteparin & 2,500 & 22 \\
& 5,000 & 40 \\
& 7,500 & 1 \\
Enoxaparin & 4,000 & 56 \\
& 6,000 & 46 \\
Heparin calcium & 8,000 & 3 \\
No therapy & $12,500 / 2$ days & 6 \\
\hline
\end{tabular}

B, Prostate procedures $(n=197)$

\begin{tabular}{lcc}
\hline Prophylaxis & Doses (IU) & $\mathrm{N}$ \\
\hline Dalteparin & 2,500 & 22 \\
& 5,000 & 58 \\
Enoxaparin & 4,000 & 81 \\
& 6,000 & 27 \\
Heparin calcium & 8,000 & 4 \\
& $12,500 / 2$ days & 1 \\
Nadroparin & 12,500 & 2 \\
No therapy & $0.6^{\mathrm{a}} / 2$ days & 1 \\
\hline
\end{tabular}

C, Bladder procedures $(\mathrm{n}=55)$

\begin{tabular}{lcr}
\hline Prophylaxis & Doses (IU) & $\mathrm{N}$ \\
\hline Dalteparin & 2,500 & 4 \\
& 5,000 & 22 \\
Enoxaparin & 4,000 & 19 \\
& 6,000 & 7 \\
Heparin calcium & 8,000 & 2 \\
& $5,000 / 2$ days & 1
\end{tabular}

$\mathrm{D}$, Miscellaneous $(\mathrm{n}=51)$

\begin{tabular}{lcr}
\hline Prophylaxis & Doses (IU) & $\mathrm{N}$ \\
\hline Dalteparin & 2,500 & 9 \\
& 5,000 & 11 \\
Enoxaparin & 4,000 & 17 \\
& 6,000 & 6 \\
& 8,000 & 1 \\
Heparin calcium & $5,000 / 3$ days & 1 \\
Nadroparin & 2,850 & 1 \\
No therapy & - & 5 \\
\hline
\end{tabular}

${ }^{\mathrm{a}}$ Units are in $\mathrm{ml}$, not IU.

surgery for cancer, and a lymphadenectomy was performed in $38.4 \%(174 / 453)$ of the total cases. The mean duration of 
surgery was $159.08 \pm 68.57 \mathrm{~min}$, and $10.6 \%$ of the patients received intraoperative transfusions. The time to flatus and to defecation was $2.19 \pm 0.98$ and $3.45 \pm 1.23$ days, respectively.

Parenteral nutrition was required for $9.3 \%$ of the patients, and 50 patients $(11.0 \%)$ needed a postoperative blood transfusion. Re-intervention was performed in 32 cases $(7.1 \%)$ due to the occurrence of grade $\geq 3$ postoperative complications (Table IV); 9 patients $(2.0 \%)$ were re-hospitalised within 90 days of the procedure.

Postoperative complications. In accordance with the Clavien-Dindo classification (12), 35.5\% (161/453) of the patients did not exhibit any postoperative complications; 163 patients $(36.0 \%)$ experienced grade 1 complications, 88 (19.4\%) experienced grade 2 complications, 27 (6.0\%) experienced grade 3 complications, 9 (2.0\%) experienced grade 4 complications and $3(0.7 \%)$ experienced grade 5 complications (deceased). Overall, 8.6\% of the patients experienced postoperative complications of Clavien-Dindo grade $\geq 3$ (Table IV), among which only 1 case was due to thromboembolism $(0.2 \%)$.

Risk of postoperative blood transfusion. Univariate analysis showed that patients who had undergone intraoperative transfusions $(\mathrm{P}=0.022)$, were aged $\geq 65$ years $(\mathrm{P}=0.009)$, exhibited multiple comorbidities $(\mathrm{P}=0.002)$, had an ASA score of $\geq 3$ $(\mathrm{P}=0.004)$ or had been treated with $>4,000$ IU enoxaparin $(\mathrm{P}=0.045)$ were at a significantly higher risk of postoperative blood transfusion. Based on multivariate analysis, only comorbidities were found to be significantly associated with a higher risk of postoperative blood transfusion $(\mathrm{P}=0.038)$ (Table V).

Risk of re-intervention. Univariate analysis showed that the risk of re-intervention was significantly higher in patients who had received intraoperative blood transfusions $(\mathrm{P}=0.006)$, were male $(\mathrm{P}=0.028)$, had undergone bladder surgery $(\mathrm{P}=0.004)$ or lymphadenectomy $(\mathrm{P}=0.031)$, exhibited comorbidities $(\mathrm{P}=0.040)$, had an ASA score of $\geq 3(\mathrm{P}=0.023)$ or had received $>4,000$ IU enoxaparin $(\mathrm{P}=0.001)$. Multivariate analysis confirmed antithrombotic prophylaxis with $>4,000$ IU enoxaparin $(\mathrm{P}=0.013)$, bladder surgery $(\mathrm{P}=0.004)$ and intraoperative transfusions $(\mathrm{P}=0.024)$ as independent risk factors for re-intervention (Table VI). A prolonged hospital stay $(\mathrm{P}<0.001)$, mobilisation $(\mathrm{P}=0.002)$ and a prolonged duration of surgery $(\mathrm{P}=0.019)$ represent additional risk factors for re-intervention (multivariate analysis).

Risk of postoperative complications. Univariate analysis showed that patients who were male $(\mathrm{P}=0.026)$, had undergone bladder surgery ( $\mathrm{P}=0.007)$, were aged $\geq 65$ years $(\mathrm{P}=0.027)$, exhibited comorbidities $(\mathrm{P}=0.013)$, had an ASA score of $\geq 3(\mathrm{P}=0.001)$ or had received prophylaxis with $>4,000 \mathrm{IU}$ enoxaparin $(\mathrm{P}<0.001)$ were at a higher risk of developing complications of grade $\geq 3$. Based on multivariate analysis, prophylaxis with $>4,000$ IU enoxaparin $(\mathrm{P}=0.002)$, bladder surgery $(\mathrm{P}=0.008)$ and an ASA score of $\geq 3(\mathrm{P}=0.037)$ were associated with a higher complication risk (Table VII).

\section{Discussion}

Deep vein thrombosis (DVT) associated with pulmonary thromboembolism is a common post-surgical urological
Table IV. Complications.

\begin{tabular}{|c|c|c|}
\hline Procedure & Clavien-Dindo grade & $\mathrm{n}(\%)$ \\
\hline \multirow[t]{8}{*}{ Prostate, $n=197$} & I & $67(34.0)$ \\
\hline & II & $23(11.3)$ \\
\hline & IIIa & $2(1.0)$ \\
\hline & IIIb & $14(7.1)$ \\
\hline & IVa & $4(2.0)$ \\
\hline & $\mathrm{IVb}$ & $0(0.0)$ \\
\hline & $\mathrm{V}$ & $0(0.0)$ \\
\hline & $\mathrm{d}$ & $0(0.0)$ \\
\hline \multicolumn{3}{|l|}{ Bladder, $\mathrm{n}=55$} \\
\hline & I & $13(23.6)$ \\
\hline & II & $26(47.3)$ \\
\hline & IIIa & $1(1.8)$ \\
\hline & IIIb & $5(9.1)$ \\
\hline & Iva & $0(0.0)$ \\
\hline & $\mathrm{IVb}$ & $2(3.6)$ \\
\hline & $\mathrm{V}$ & $2(3.6)$ \\
\hline & $\mathrm{d}$ & $0(0.0)$ \\
\hline \multicolumn{3}{|l|}{ Kidney, $n=150$} \\
\hline & $\mathrm{I}$ & $63(42.0)$ \\
\hline & II & $33(22.0)$ \\
\hline & IIIa & $1(0.7)$ \\
\hline & IIIb & $1(0.7)$ \\
\hline & IVa & $1(0.7)$ \\
\hline & $\mathrm{IVb}$ & $0(0.0)$ \\
\hline & $\mathrm{V}$ & $1(0.7)$ \\
\hline & $\mathrm{d}$ & $0(0.0)$ \\
\hline \multicolumn{3}{|c|}{ Miscellaneous, $\mathrm{n}=51$} \\
\hline & $\mathrm{I}$ & $19(37.3)$ \\
\hline & II & $8(15.7)$ \\
\hline & IIIa & $0(0.0)$ \\
\hline & IIIb & $3(5.9)$ \\
\hline & IVa & $2(3.9)$ \\
\hline & $\mathrm{IVb}$ & $0(0.0)$ \\
\hline & $\mathrm{V}$ & $0(0.0)$ \\
\hline & $\mathrm{d}$ & $0(0.0)$ \\
\hline
\end{tabular}

complication. Furthermore, pulmonary thromboembolism is the most common cause of mortality in non-surgical urology (1), also constituting a significant risk factor for postoperative morbidity (13). Consequently, the prevention of thromboembolism represents an important strategic objective for the reduction of postoperative mortality and morbidity. Despite this fact, urological guidelines have been based on a low level of evidence, in the absence of randomised controlled trials (4).

Patients can be divided into four groups, with regard to the post-surgical thromboembolic risk: Low, moderate, high and very high. Virtually all patients undergoing open urological surgery are at risk of developing DVT and pulmonary embolism. Each patient may exhibit a number of clinical risk factors for thromboembolism, such as advanced age, obesity, varicose veins, previous VTE, hereditary and acquired thrombophilia, other thrombotic states, malignancy, hormone therapy, pregnancy, childbirth, immobilisation, central venous catheter and lower-limb injuries (2). 
Table V. Uni- and multivariate analysis of postoperative transfusion risk.

\begin{tabular}{|c|c|c|c|c|c|}
\hline \multirow[b]{2}{*}{ Risk factor } & \multirow[b]{2}{*}{$\mathrm{N}$} & \multicolumn{2}{|c|}{ Postoperative transfusion } & \multirow{2}{*}{$\begin{array}{c}\text { Univariate analysis } \\
\text { P-value }\end{array}$} & \multirow{2}{*}{$\begin{array}{c}\text { Multivariate analysis } \\
\text { P-value }\end{array}$} \\
\hline & & Yes, n $(\%)$ & No, n $(\%)$ & & \\
\hline Intraoperative transfusion & & & & 0.022 & n.s. \\
\hline Yes & 48 & $10(20.8)$ & $38(79.2)$ & & \\
\hline No & 405 & $40(9.9)$ & $365(90.1)$ & & \\
\hline Age $\geq 65$ years & & & & 0.009 & n.s. \\
\hline Yes & 238 & $35(14.7)$ & $203(85.3)$ & & \\
\hline No & 215 & $15(7.0)$ & $200(93.0)$ & & \\
\hline Comorbidity & & & & 0.002 & 0.038 \\
\hline Yes & 361 & $48(13.3)$ & $313(86.7)$ & & \\
\hline No & 90 & $2(2.2)$ & $90(97.8)$ & & \\
\hline ASA score $\geq 3$ & & & & 0.004 & n.s. \\
\hline Yes & 108 & $20(18.5)$ & $88(81.5)$ & & \\
\hline No & 340 & $29(8.5)$ & $311(91.5)$ & & \\
\hline Enoxaparin $>4,000 \mathrm{IU}$ & & & & 0.045 & n.s. \\
\hline Yes & 66 & $12(18.2)$ & $54(81.8)$ & & \\
\hline Other & 387 & $38(9.8)$ & $349(10.2)$ & & \\
\hline
\end{tabular}

ASA, American Society of Anaesthesiologists; n.s., not significant.

Table VI. Uni- and multivariate analysis of postoperative re-intervention risk.

\begin{tabular}{|c|c|c|c|c|c|}
\hline \multirow[b]{2}{*}{ Risk factor } & \multirow[b]{2}{*}{$\mathrm{N}$} & \multicolumn{2}{|c|}{ Re-intervention } & \multirow{2}{*}{$\begin{array}{c}\text { Univariate analysis } \\
\text { P-value }\end{array}$} & \multirow{2}{*}{$\begin{array}{c}\text { Multivariate analysis } \\
\text { P-value }\end{array}$} \\
\hline & & Yes, n $(\%)$ & No, n $(\%)$ & & \\
\hline Comorbidity & & & & 0.040 & n.s. \\
\hline Yes & 361 & $30(8.3)$ & $331(91.7)$ & & \\
\hline No & 92 & $2(2.2)$ & $90(97.8)$ & & \\
\hline $\mathrm{ASA} \geq 3$ & & & & 0.023 & n.s. \\
\hline Yes & 108 & $19(17.6)$ & $321(82.4)$ & & \\
\hline No & 340 & $13(3.8)$ & $95(96.2)$ & & \\
\hline Intraoperative transfusion & & & & 0.006 & 0.024 \\
\hline Yes & 48 & $8(16.7)$ & $40(83.3)$ & & \\
\hline No & 405 & $24(5.9)$ & $381(94.1)$ & & \\
\hline Male gender & & & & 0.028 & n.s. \\
\hline Yes & 355 & $30(8.5)$ & $325(91.5)$ & & \\
\hline No & 98 & $2(2.0)$ & $96(98.0)$ & & \\
\hline Bladder surgery & & & & 0.004 & 0.004 \\
\hline Yes & 55 & $9(16.4)$ & $46(83.6)$ & & \\
\hline No & 398 & $23(5.8)$ & $375(94.2)$ & & \\
\hline Lymphadenectomy & & & & 0.031 & n.s. \\
\hline Yes & 174 & $18(10.3)$ & $156(89.7)$ & & \\
\hline No & 279 & $14(5.0)$ & $265(95.0)$ & & \\
\hline Enoxaparin $>4,000 \mathrm{IU}$ & & & & 0.001 & 0.013 \\
\hline Yes & 66 & $11(16.7)$ & $55(83.3)$ & & \\
\hline Other & 387 & $21(5.4)$ & $366(94.6)$ & & \\
\hline
\end{tabular}

ASA, American Society of Anaesthesiologists; n.s., not significant.

In the present study, $98 \%$ of the patients received both mechanical and pharmacological prophylaxis for VTE, and only 1 case of proximal DVT, which was not associated with pulmonary thromboembolism, was observed; in 2 other cases the clinical suspicion of distal DVT was not radiologically confirmed. 
Table VII. Uni- and multivariate analysis of high postoperative complication risk.

\begin{tabular}{|c|c|c|c|c|c|}
\hline \multirow[b]{2}{*}{ Risk factor } & \multirow[b]{2}{*}{$\mathrm{N}$} & \multicolumn{2}{|c|}{ Re-intervention } & \multirow{2}{*}{$\begin{array}{c}\text { Univariate analysis } \\
\text { P-value }\end{array}$} & \multirow{2}{*}{$\begin{array}{c}\text { Multivariate analysis } \\
\text { P-value }\end{array}$} \\
\hline & & Yes, n (\%) & No, n $(\%)$ & & \\
\hline Comorbidity & & & & 0.013 & n.s. \\
\hline Yes & 361 & $37(10.2)$ & $324(89.8)$ & & \\
\hline No & 92 & $2(2.2)$ & $90(97.8)$ & & \\
\hline $\mathrm{ASA} \geq 3$ & & & & 0.001 & 0.037 \\
\hline Yes & 108 & $18(16.7)$ & $90(83.3)$ & & \\
\hline No & 340 & $21(6.2)$ & $317(93.8)$ & & \\
\hline Age $>65$ years & & & & 0.027 & n.s. \\
\hline Yes & 238 & $27(11.4)$ & $209(88.6)$ & & \\
\hline No & 215 & $12(5.6)$ & $203(94.4)$ & & \\
\hline Male gender & & & & 0.026 & n.s. \\
\hline Yes & 355 & $36(10.1)$ & $317(89.9)$ & & \\
\hline No & 98 & $3(3.1)$ & $95(96.9)$ & & \\
\hline Bladder surgery & & & & 0.007 & 0.008 \\
\hline Yes & 55 & $10(18.2)$ & $45(81.8)$ & & \\
\hline No & 396 & $29(7.3)$ & $367(96.7)$ & & \\
\hline Enoxaparin $>4,000 \mathrm{IU}$ & & & & $<0.001$ & 0.002 \\
\hline Yes & 66 & $15(22.7)$ & $51(87.3)$ & & \\
\hline Other & 387 & $24(6.2)$ & $361(93.8)$ & & \\
\hline
\end{tabular}

ASA, American Society of Anaesthesiologists; n.s., not significant.

During the study period, several antithrombotic protocols were administered, using different types of LWMH, depending on the cardiovascular comorbidity of the patients. Two types of LWMH were predominantly used (Table III): Between December 2011 and September 2012, subcutaneous (s.c.) dalteparin was used, whereas between September 2012 and March 2013, enoxaparin was used. The most commonly administered dalteparin dosage was 5,000 IU s.c. (131/189 patients); however, a lower dose of 2,500 IU dalteparin was used in 58 low-risk patients. With regard to enoxaparin, a dosage of 4,000 IU s.c. was administered to 173 patients, whereas 67 patients received a higher dose $(6,000$ or $8,000 \mathrm{IU})$. Higher doses were necessary in patients with a BMI of $>30 \mathrm{~kg} / \mathrm{m}^{2}$ or those who were considered to be at a high risk of venous thromboembolism, including some already undergoing anticoagulant therapy. An increase in dose was often necessary, as the majority of the patients exhibited several comorbidities, particularly metabolic disorders and cardiovascular diseases: Arterial hypertension in $50.1 \%$ of the cases (227 patients), diabetes in $11.0 \%$ (50 patients), ischemic heart disease in $9.7 \%$ (44 patients) and chronic atrial fibrillation in $4.0 \%$ (18 patients). Only 92 patients $(20.3 \%)$ were free from comorbidities at the time of surgery.

The prophylaxis protocols used in the present study were almost overlapping with those recommended by the AUA (4). In almost all cases, heparin administration was postoperatively prolonged for 3 or 4 weeks, in accordance with the ACCP guidelines, since this leads to a reduction in the rate of venous thromboembolism without significantly affecting the occurrence of complications (13).
The prophylaxis for VTE used in the present study was effective in reducing the risk of thromboembolism; no cases of pulmonary embolism developed and only 1 patient presented proximal DVT, confirming the data from previous studies on thromboembolism prophylaxis (4); however, a significant correlation between the use of enoxaparin doses $>4,000$ IU and the occurrence of major complications (Clavien-Dindo $\geq 3$ ) and re-intervention was found. Approximately one-half of the re-interventions were performed due to haemorrhagic complications (haematoma), suggesting that, compared with other LMWHs, high-dose enoxaparin can lead to a higher rate of bleeding, particularly during bladder surgery, which is the more complex branch of urological surgery.

In this retrospective analysis of 453 consecutive patients undergoing urological surgery, a complication rate of $64.5 \%$ (292/453 patients) was documented. According to the Clavien-Dindo classification, serious complications (Clavien-Dindo $\geq 3$ ) occurred only in 39 cases $(8.6 \%)$.

The thromboembolism prophylaxis protocol used in the present study was found to be effective, as no patient developed severe thromboembolic complications and only 1 patient developed VTE; however, the results indicated that high-dose enoxaparin (>4,000 IU) should not be used for thromboembolism prophylaxis, as it represents an independent risk factor, together with bladder surgery, for major complications and re-intervention.

\section{References}

1. Kibel AS and Loughlin KR: Pathogenesis and prophylaxis of postoperative thromboembolic disease in urological pelvic surgery. J Urol 153: 1763-1764, 1995. 
2. Geerts WH, Pineo GF, Heit JA, Bergqvist D, Lassen MR, Colwell CW and Ray JG: Prevention of venous thromboembolism: The Aeventh ACCP Conference on Antithrombotic and Thrombolytic Therapy. Chest 126 (Suppl 3): 338S-400S, 2004.

3. White RH, Zhou H and Romano PS: Incidence of symptomatic venous thromboembolism after different elective or urgent surgical procedures. Thromb Haemost 90: 446-455, 2003.

4. Forrest JB, Clemens JQ, Finamore P, Leveillee R, Lippert M, Pisters L, Touijer K and Whitmore K; American Urological Association: AUA best practice statement for the prevention of deep vein thrombosis in patients undergoing urologic surgery. J Urol 181: 1170-1177, 2009.

5. Geerts WH, Bergqvist D, Pineo GF, Heit JA, Samama CM, Lassen MR and Colwell CW; American College of Chest Physicians: Prevention of venous thromboembolism: American College of Chest Physicians Evidence-Based Clinical Practice Guidelines (8th edition). Chest 133 (Suppl 6): 381S-453S, 2008.

6. Secin FP, Jiborn T, Bjartell AS, Fournier G, Salomon L, Abbou CC, Haber GP, Gill IS, Crocitto LE, Nelson RA, et al: Multi-institutional study of symptomatic deep venous thrombosis and pulmonary embolism in prostate cancer patients undergoing laparoscopic or robot-assisted laparoscopic radical prostatectomy. Eur Urol 53: 134-145, 2008.
7. Rice KR, Brassell SA and McLeod DG: Venous thromboembolism in urologic surgery: Prophylaxis, diagnosis and treatment. Rev Urol 12: e111-e124, 2010.

8. Goossens-Laan CA, Visser O, Hulshof MC, Wouters MW, Bosch JL, Coebergh JW and Kil PJ: Survival after treatment for carcinoma invading bladder muscle: A Dutch population-based study on the impact of hospital volume. BJU Int 110: 226-232, 2012.

9. Shabsigh A, Korets R, Vora KC, Brooks CM, Cronin AM Savage C, Raj G, Bochner BH, Dalbagni G, Herr HW and Donat SM: Defining early morbidity of radical cystectomy for patients with bladder cancer using a standardized reporting methodology. Eur Urol 55: 164-174, 2009.

10. Charlson M, Szatrowski TP, Peterson J and Gold J: Validation of a combined comorbidity index. J Clin Epidemiol 47: 1245-1251, 1994.

11. Dripps Rd, Lamont A and Eckenhoff JE: The role of anesthesia in surgical mortality. JAMA 178: 261-266, 1961.

12. Dindo D, Demartines N and Clavien PA: Classification of surgical complications: A new proposal with evaluation in a cohort of 6336 patients and results of a survey. Ann Surg 240: 205-213, 2004

13. Franzeck UK, Schalch I, Jäger KA, Schneider E, Grimm J and Bollinger A: Prospective 12 year follow up study of clinical and hemodynamic sequelae of deep vein thrombosis in low risk patients (Zürich study). Circulation 93: 74-79, 1996. 